\title{
Adhesion Layer Influence on Controlling the Local Temperature in Plasmonic Gold
} Nanoholes

Quanbo Jiang, Benoît Rogez, Jean-Benoît Claude, Antonin Moreau, Julien Lumeau, Guillaume Baffou, Jérôme Wenger*

Aix Marseille Univ, CNRS, Centrale Marseille, Institut Fresnel, 13013 Marseille, France

* Corresponding author: jerome.wenger@fresnel.fr

\begin{abstract}
Gold films do not adhere well on glass substrates, so plasmonics experiments typically use a thin adhesion layer of titanium or chromium to ensure a proper adhesion between the gold film and the glass substrate. While the absorption of light into gold structures is largely used to generate heat and control the temperature at the nanoscale, the influence of the adhesion layer on this process is largely overlooked. Here, we quantify the role of the adhesion layer in determining the local temperature increase around a single nanohole illuminated by a focused infrared laser. Despite their nanometer thickness, adhesion layers can absorb a greater fraction of the incoming infrared light than the $100 \mathrm{~nm}$ thick gold layer leading to a significant increase of the local temperature. Different experimental designs are explored, offering new ways to promote or avoid the temperature increase inside nanoapertures. This knowledge further expands the plasmonic toolbox for temperature-controlled experiments including single molecule sensing, nanopore translocation, polymerization, or nano-optical trapping.
\end{abstract}
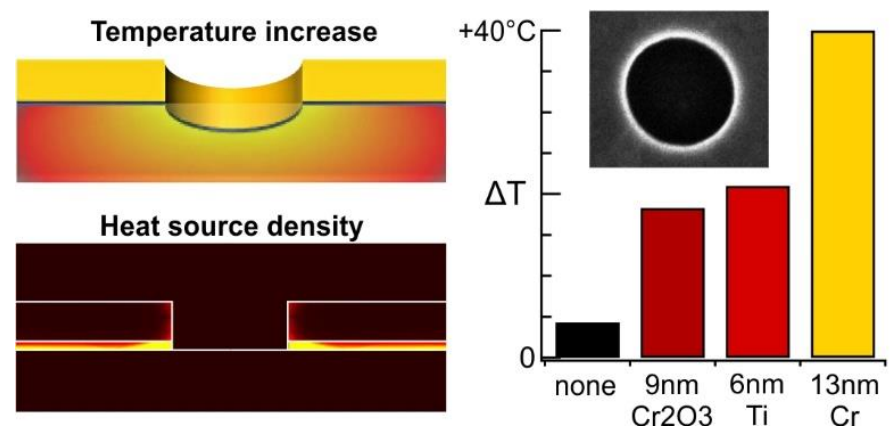

Figure for Table of Contents

Keywords : thermoplasmonics, nanoaperture, zero-mode waveguide, temperature measurements, nanooptical trapping 


\section{Introduction}

While plasmonics offers many opportunities to control the light fields into nanoscale dimensions, it is also intrinsically bound to the occurrence of absorption and Ohmic losses into the metal. ${ }^{1}$ These losses eventually lead to Joule heating of the surrounding environment, ${ }^{2}$ hereby increasing the local temperature. This new ability to generate local temperature distributions has driven the emergence of thermoplasmonics to control heat generation at the nanoscale, ${ }^{3-5}$ which is fruitful for various applications in different fields of physics, biology and chemistry. ${ }^{6-8}$

Evaporated gold films, which are commonly used in plasmonics, generally require the presence of an additional adhesion layer (typically a few nanometers of titanium or chromium) to promote the adhesion of the gold structure onto the glass substrate. ${ }^{9,10}$ Despite its small thickness in the nanometer range, this adhesion layer may have a significant influence on the plasmonic response of the gold structure. For instance, the adhesion layer was shown to shift and broaden the plasmon resonance, ${ }^{11-14}$ affect the transmission through nanohole arrays, ${ }^{15}$ damp the enhancement factor in surface-enhanced fluorescence ${ }^{16,17}$ and Raman scattering, ${ }^{18-20}$ and affect the nano-optical trapping potential. ${ }^{21}$ However, in the context of heat generation in thermoplasmonics, the role of this supplementary adhesion layer remains largely unexplored and very little is known about the quantitative influence of various gold adhesion layers in the temperature increase around illuminated gold nanostructures. ${ }^{22}$

Here, we experimentally and numerically quantify the role of the gold adhesion layer in determining the local temperature increase around a single nanohole (SNH) illuminated by a focused infrared laser beam. We also investigate supplementary approaches to control the temperature by properly selecting the illumination geometry and the substrate material. Nanoholes are chosen to benchmark the influence of the adhesion layer, as these structures have received a large interest owing to their ability to confine light into attoliter volumes, ${ }^{23,24}$ enhance the luminescence of single quantum emitters, ${ }^{16,25}$ and trap single nano-objects. ${ }^{26,27}$ Our experimental configuration reproduces the typical arrangement used for nanooptical trapping using nanoholes, ${ }^{27-35}$ and therefore our results can be directly applied to these experiments. The choice of a single nanohole of fixed diameter is not a limitation: we have recently shown that the temperature increases inside a single or a double nanohole are quite comparable and largely independent of the plasmonic resonance for aperture diameters below $350 \mathrm{~nm} .{ }^{36}$ Thus, the results reported here for a $300 \mathrm{~nm}$ diameter SNH directly apply also to other nano-optical trapping experiments. ${ }^{27-35}$ More generally, adjusting the adhesion layer and the experimental configuration bring new options to control the local temperature with plasmonics. This further expands the 
thermoplasmonics toolbox for several heat-controlled applications including polymerization, ${ }^{37}$ enzymatic activity monitoring, ${ }^{23,24}$ nanopore translocation, ${ }^{38-40}$ or thermophoretic trapping. ${ }^{41,42}$

a

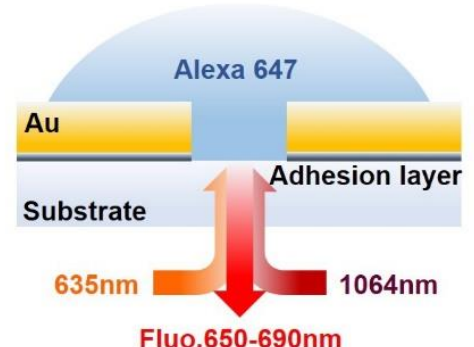

Fluo.650-690nm

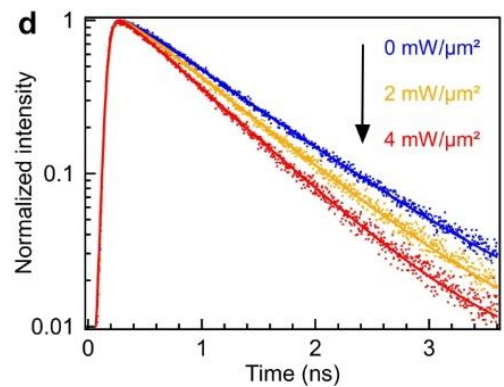

b
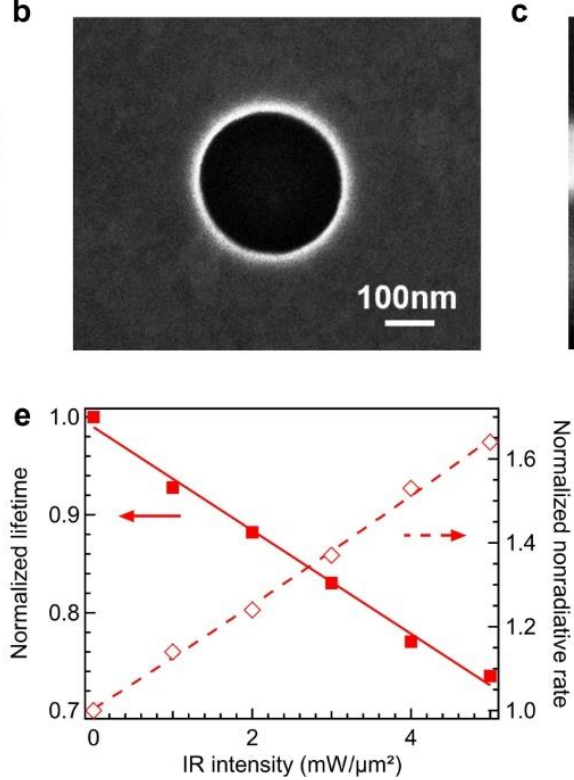

C
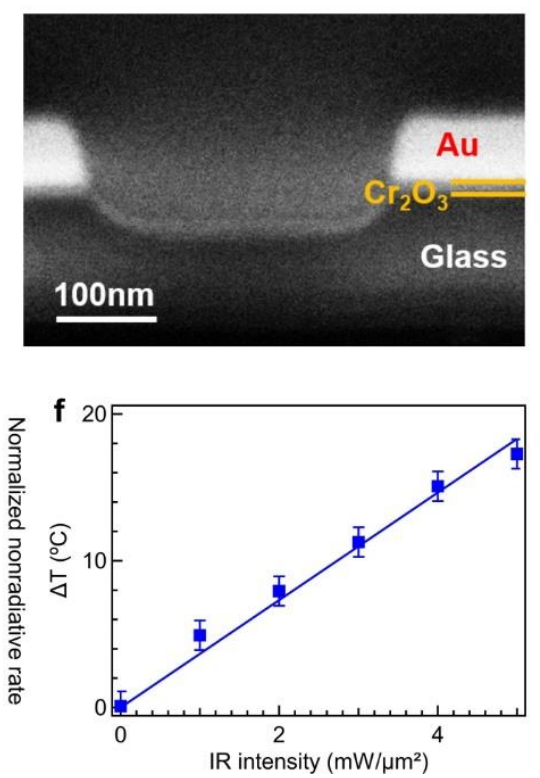

Figure 1. Temperature measurement in a single nanohole. (a) Sketch of the experimental setup with a $1064 \mathrm{~nm}$ focused laser beam to heat the nanostructure and a $635 \mathrm{~nm}$ laser beam to probe the fluorescence of Alexa Fluor 647 solution filling the nanohole. In this configuration, the adhesion layer between the gold film and the glass substrate is illuminated and heated. (b) Top view SEM image and (c) cross-section with $52^{\circ}$ tilted view SEM image of a $300 \mathrm{~nm}$ diameter aperture milled in gold. The $9 \mathrm{~nm} \mathrm{Cr}_{2} \mathrm{O}_{3}$ adhesion layer is visible between the Au film and the glass substrate. (d) Normalized fluorescence decay curves of Alexa Fluor 647 in the $300 \mathrm{~nm}$ diameter SNH with $9 \mathrm{~nm} \mathrm{Cr}_{2} \mathrm{O}_{3}$ adhesion layer recorded with 3 different IR intensities. From top to bottom, blue, orange and red curves correspond to the IR intensities of 0,2 and $4 \mathrm{~mW} / \mu \mathrm{m}^{2}$ respectively. (e) Normalized lifetime (solid curve) and nonradiative decay rate (dashed curve) deduced from the fluorescence decay curves in (d) as a function of the IR intensity. (f) Temperature increase estimated from the lifetime data in (e) as a function of the IR intensity.

\section{Results and Discussion}

Our experiments focus on a single nanohole (SNH) of $300 \mathrm{~nm}$ diameter milled by focused ion beam (FIB) in a $100 \mathrm{~nm}$ thick gold film (Fig. 1a,b). A $1064 \mathrm{~nm}$ infrared (IR) laser beam focused by a high NA microscope objective is used to heat the gold $\mathrm{SNH}$. We have recently developed a technique to measure the 
temperature inside SNH structures based on monitoring the fluorescence lifetime of Alexa Fluor 647 molecules. ${ }^{36}$ This technique has also been validated against two other independent experimental measurements based on fluorescence intensity and diffusion time, and against numerical simulations. ${ }^{36}$ The key concept in the fluorescence lifetime approach is that the nonradiative decay rate constant of the Alexa Fluor 647 dyes is temperature dependent and can be calibrated to measure locally the temperature around a SNH. The Methods section briefly summarizes the approach and main equations needed to compute back the temperature increase from the fluorescence lifetime measurements. In addition to the infrared heating laser, we overlap a $635 \mathrm{~nm}$ pulsed red laser with $20 \mu \mathrm{W} / \mu \mathrm{m}^{2}$ average intensity to excite the fluorescence and record the lifetime. We neglect the heating effect induced by the red laser as its intensity remains always small compared to the IR laser intensity in the $\mathrm{mW} / \mathrm{\mu m}^{2}$ range.

We have recently shown that the temperature increase inside SNHs does not significantly depend on the nanohole diameter as long as the diameter is below $350 \mathrm{~nm} .{ }^{36}$ Similarly, the temperature increase is quite comparable between single and double nanohole structures, independently of the plasmonic resonance, and is mainly dictated by the absorption and Joule losses into the metal layer. Therefore, the results discussed here for the $300 \mathrm{~nm}$ diameter SNH can be readily extended to other SNH diameters, and even double nanohole structures (DNH).

Titanium, chromium or chromium oxide layers are typically used in plasmonics to ensure a proper adhesion between the gold film and the glass substrate. ${ }^{11-13,15-21}$ Here we use a representative selection of these materials and test different thicknesses for the adhesion layer. For example, the $9 \mathrm{~nm}$ thick chromium oxide $\left(\mathrm{Cr}_{2} \mathrm{O}_{3}\right)$ adhesion layer can be seen by scanning electron microscopy (SEM) after half the SNH structure has been cut by FIB to show a cross-section image of the SNH (Fig. 1c).

For each adhesion layer, we record the temperature increase as a function of the IR heating intensity. Figure $1 \mathrm{~d}$-f briefly presents typical experimental results for a $9 \mathrm{~nm} \mathrm{Cr}_{2} \mathrm{O}_{3}$ adhesion layer. The starting point is the fluorescence decay histograms (Fig. 1d) measured on a $300 \mathrm{~nm}$ diameter SNH filled with the Alexa Fluor 647 water solution. The three histograms on Fig. 1d for increasing IR intensities readily show a clear fluorescence lifetime reduction as the IR intensity raises. From the fluorescence decays, we extract the fluorescence lifetime and the nonradiative decay rate which are temperature dependent (Fig. 1e). Using the calibrated temperature dependence and the technique described in the Methods section, we can compute back the temperature increase for each IR illumination intensity (Fig. 1f). For all cases, we observe a linear relationship between the temperature increase and the IR intensity, as expected from the linearity of the heat-diffusion equation. ${ }^{5}$ 

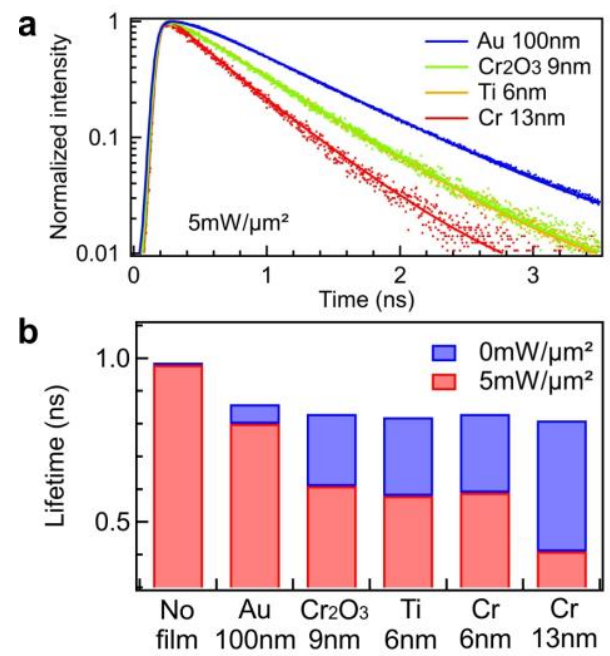

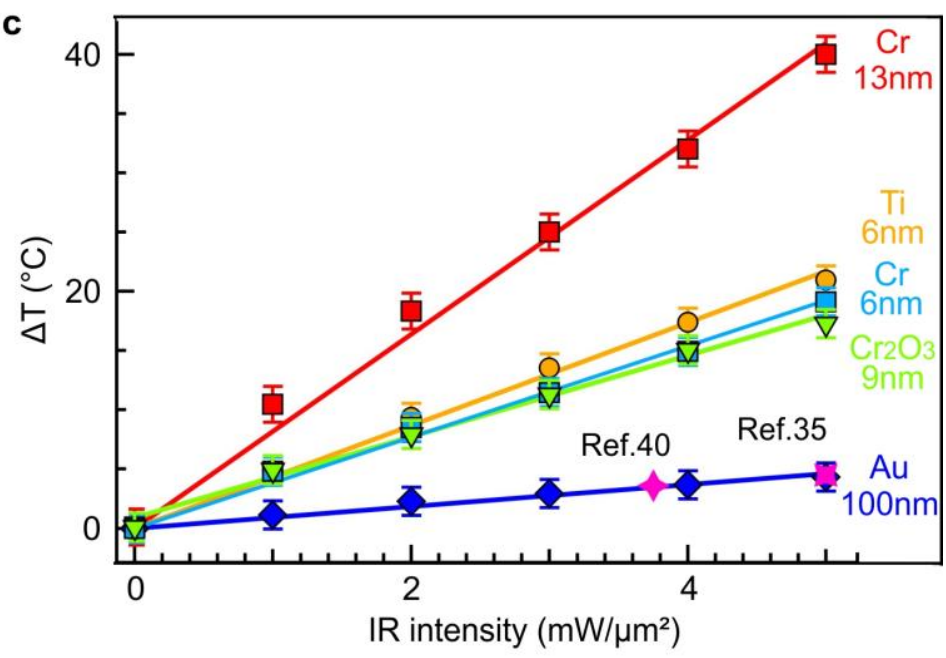

Figure 2. Influence of the adhesion layer for the gold film on the temperature increase. (a) Normalized fluorescence decay curves in $300 \mathrm{~nm}$ diameter gold SNHs with different adhesion layers at $5 \mathrm{~mW} / \mu \mathrm{m}^{2} \mathrm{IR}$ illumination. (b) Fluorescence lifetime values for different adhesion layers. The case "no film" is the reference for a glass coverslip without any metal. The blue and red bars indicate the cases without and with a $5 \mathrm{~mW} / \mu \mathrm{m}^{2} \mathrm{IR}$ illumination. (c) Temperature increase measured on a $300 \mathrm{~nm}$ SNH for different gold adhesion layers. All the other experimental conditions are identical. The pink cross markers refer to independent measurements and simulations in Ref ${ }^{40}$ and ${ }^{35}$ respectively.

The main goal of our paper is to explore how the range of temperature increase can be tuned by properly choosing the nature and thickness of the gold adhesion layer, the illumination geometry and the substrate material. Figure 2 summarizes our results for different adhesion layers. We also show the results obtained when only gold is illuminated (all other experimental parameters are identical). Although the same SNH diameter, same gold thickness, and same IR power are used, the fluorescence decay histograms recorded for different adhesion layers directly show a pronounced difference, which indicates a different temperature elevation (Fig. 2a). When no infrared beam is present, the fluorescence lifetime is reduced by the gold SNH from the $1.0 \mathrm{~ns}$ confocal value to $0.8 \pm 0.05 \mathrm{~ns}$ (Fig. 2b, blue bars). This moderate lifetime reduction is ascribed to the SNH size and quite independent of the choice of the adhesion layer, confirming that the Alexa Fluor 647 molecules experience similar nanophotonic environments. However, when the IR heating laser is turned on, the fluorescence lifetime is then clearly different depending on the adhesion 
layer material (Fig. 2b, red bars). This observation directly shows that the adhesion layer plays a key role in the local temperature increase inside a SNH.

Based on the lifetime reduction measured for each adhesion layer compared with the case without IR illumination, we compute back the temperature increase following the same procedure as for Fig. If. Figure 2c summarizes our main results. The $13 \mathrm{~nm} \mathrm{Cr}$ adhesion layer yields the highest temperature increase for a given IR power, and can reach a gain up to $+40^{\circ} \mathrm{C}$ at $5 \mathrm{~mW} / \mu \mathrm{m}^{2}$ IR illumination. Here we have used a relatively thick adhesion layer in order to see how large the temperature increase can be despite the presence of the gold layer acting as a heat sink. ${ }^{43}$ Conversely, the gold film without any adhesion layer features a ten-fold reduced temperature gain with only $+4.3^{\circ} \mathrm{C}$ at the same $5 \mathrm{~mW} / \mu \mathrm{m}^{2} \mathrm{IR}$ intensity. This impressive difference reveals the major role played by the adhesion layer in controlling the local temperature increase upon SNH laser illumination. Additionally, our measurements for the gold film with no adhesion layer match very well two completely independent temperature assessments based on ionic current measurements ${ }^{40}$ or numerical simulations ${ }^{35}$ (pink cross markers in Fig. 2c). This further validates our experimental results and methodology.

Comparing between the different adhesion layers, we find that the temperature increases with $6 \mathrm{~nm} \mathrm{Ti}$ and $6 \mathrm{~nm} \mathrm{Cr}$ layer are about twice less than with $13 \mathrm{~nm} \mathrm{Cr}$ case. This effect is mainly a consequence of the reduced thickness used, as the infrared absorption of $\mathrm{Cr}$ and Ti layers at $1064 \mathrm{~nm}$ are very similar (Supporting Information Fig. S1 and S2). Furthermore, for thicknesses below $20 \mathrm{~nm}$, the IR absorption and the temperature increase are linearly proportional to the adhesion layer thickness (Supporting Information Fig. S1). Chromium oxide $\mathrm{Cr}_{2} \mathrm{O}_{3}$ is significantly less absorbing the IR than pure metals like $\mathrm{Cr}$ or Ti. Therefore, despite its $9 \mathrm{~nm}$ thickness, the temperature increase with the $\mathrm{Cr}_{2} \mathrm{O}_{3}$ layer remains slightly lower than with a $6 \mathrm{~nm} \mathrm{Ti}$ or $\mathrm{Cr}$ layer (Fig. 2c). Altogether, these experimental results show that the temperature increase inside a SNH can be largely controlled by the material chosen for the gold adhesion layer, its thickness and the IR intensity used. 


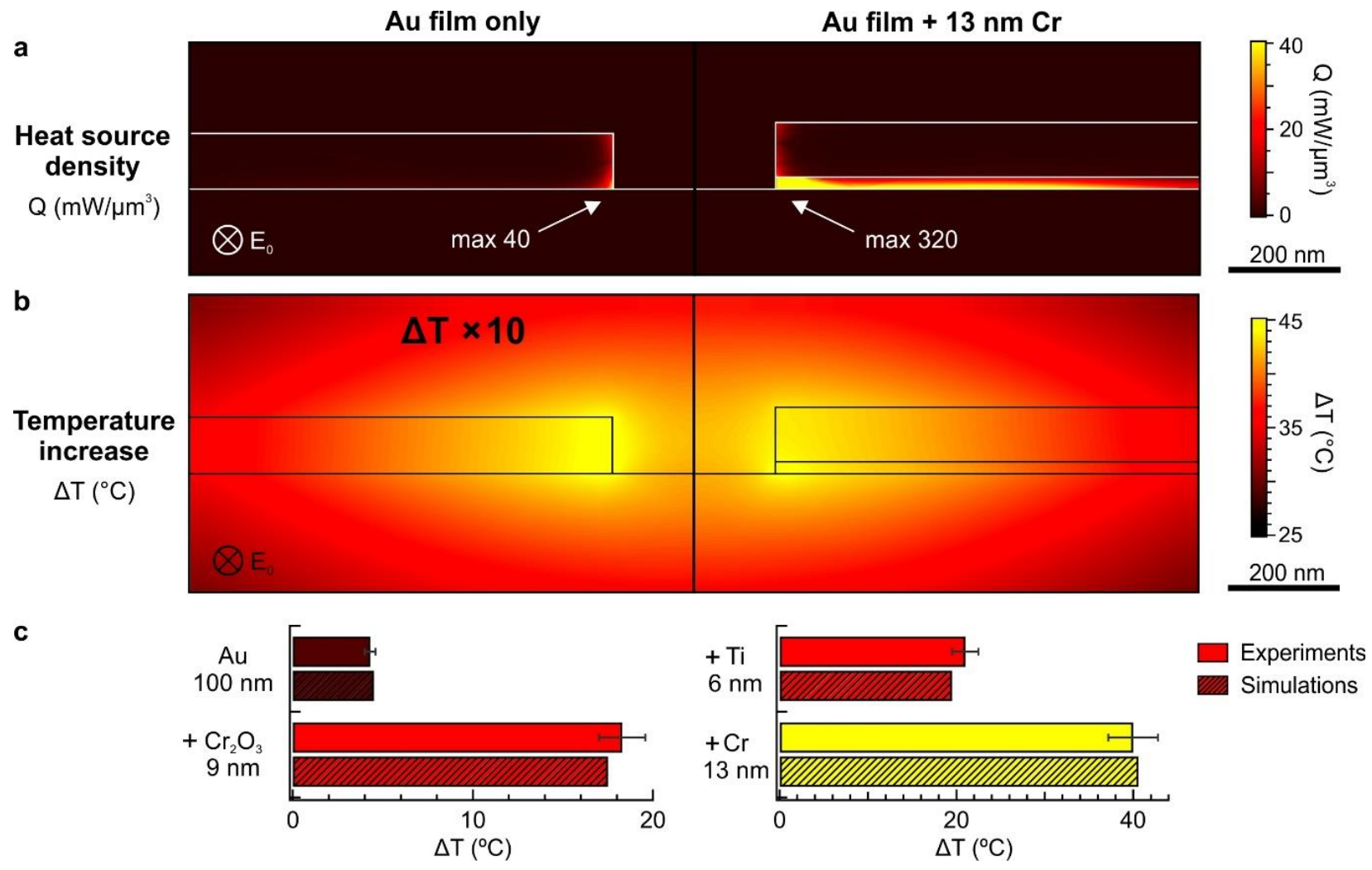

Figure 3. Numerical simulations confirming the role of the adhesion layer on the temperature increase. $(a, b)$ Spatial maps of the heat source density (a) and the temperature increase (b) around a $300 \mathrm{~nm}$ diameter gold SNH. The left part of the images shows the case without any adhesion layer (gold directly on glass substrate), while in the right part includes a $13 \mathrm{~nm}$ Cr layer between the Au film and the glass substrate. In both cases, the IR intensity is $5 \mathrm{~mW} / \mu \mathrm{m}^{2}$ with the polarization perpendicular to the plane. In (b), the temperature increase with no adhesion layer is multiplied by $10 \times$ to display on the same color scale as with the $\mathrm{Cr}$ adhesion layer. (c) Comparison of the temperature increase for different adhesion layers at $5 \mathrm{~mW} / \mu \mathrm{m}^{2} \mathrm{IR}$ intensity: the solid top bar corresponds to the experimental measurements while the bottom dashed bar shows the simulations results.

Our experimental findings suggest that the temperature increase is mainly ascribed to the IR Ohmic losses into the adhesion layer instead of the gold layer. This is quite surprising given the small thickness used for the adhesion layers (below $13 \mathrm{~nm}$ ) as compared to the $100 \mathrm{~nm}$ thick gold film. To confirm this effect, we have conducted a series of numerical simulations using COMSOL Multiphysics (Fig. 3). Spatial maps of the heat source density and temperature increase on a $300 \mathrm{~nm}$ gold SNH without (left) and with the $13 \mathrm{~nm} \mathrm{Cr}$ adhesion layer (right) are presented in Fig. 3a,b respectively. In the absence of the adhesion layer, the 
heat source essentially accumulates at the aperture edges, where the power dissipated by the laserinduced electron current is maximum. ${ }^{44}$ In the presence of the $13 \mathrm{~nm} \mathrm{Cr}$ layer, the absorption into the thin $\mathrm{Cr}$ layer generates a dominating additional contribution to the heat source (Fig. 3a). The maximum heat source density is increased by 8 fold, and now spreads over a much larger area. As a consequence of the Joule heating, the temperature rises in the nanohole. Due to the large difference of heat source densities between the cases with and without adhesion layers, the temperature increase in the nanohole with the $13 \mathrm{~nm} \mathrm{Cr}$ layer is almost ten times higher than the case without any adhesion layer (Fig. 3b), in excellent agreement with our experimental results (Fig. 2c). In both cases (with and without the adhesion layer), the $100 \mathrm{~nm}$ thick gold film serves as a heat sink to dissipate the temperature. Therefore the thermal gradients in both cases follow very similar spatial distributions, despite the ten-fold reduced amplitude in the absence of $\mathrm{Cr}$ layer.

The same numerical simulations are performed to reproduce the other experimental configurations, and the temperature increase is averaged over the SNH volume. This allows a direct comparison with the experimental results (Fig. 3c), for which we find an excellent agreement for all the different cases. This set of simulations clearly confirms that the nature and thickness of the gold adhesion layer largely control the temperature inside nanohole structures.
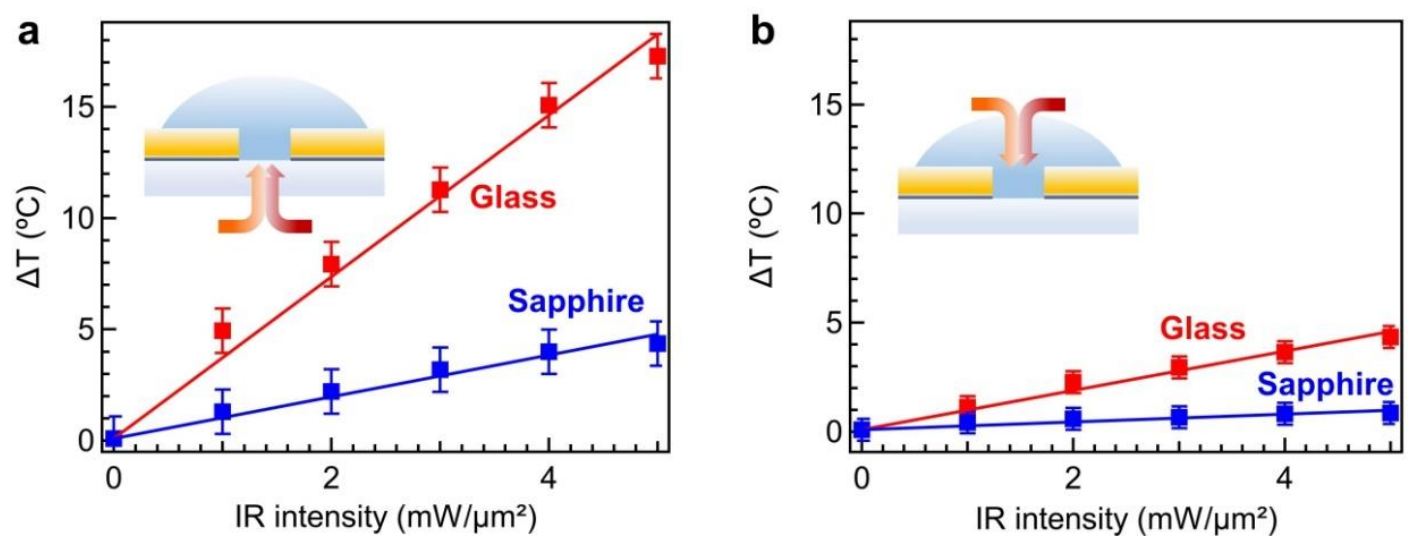

Figure 4. Comparison of the temperature increase with different illumination geometries and substrates. (a,b) Temperature increase inside a $300 \mathrm{~nm}$ gold SNH deposited on a glass (red markers) or sapphire (blue) substrate. In (a), a $9 \mathrm{~nm} \mathrm{Cr}_{2} \mathrm{O}_{3}$ adhesion layer is illuminated by the IR beam, while in (b) the IR light is incoming from the top gold surface and does not reach the adhesion layer underneath the $100 \mathrm{~nm}$ gold film. 
Other approaches can be used to further tune the temperature gain inside the SNH. Instead of the borosilicate glass, the metal layers can be deposited on a sapphire substrate, which features a much better thermal conductivity than standard glass. This introduces a supplementary heat dissipation channel, still choosing a transparent thermally-conductive substrate. Figure 4a shows our experimental results for a $300 \mathrm{~nm}$ diameter $\mathrm{SNH}$ milled in a $100 \mathrm{~nm}$ gold film with $9 \mathrm{~nm} \mathrm{Cr} 2 \mathrm{O}_{3}$ adhesion layer. The only difference is the borosilicate glass or sapphire used for the $150 \mu \mathrm{m}$ thick substrate material. In the case of sapphire, the supplementary heat dissipation brought by the substrate yields a four times lower temperature increase than with a glass coverslip.

To further damp the temperature elevation, the sample can be reversed to illuminate the gold film from the side opposite to the adhesion layer (Fig. 4b). In this case the temperature gain is similar to the case of the gold film without any adhesion layer (as the adhesion layer is no longer directly illuminated by the infrared beam). Again, we find that the use of the sapphire substrate reduces the temperature increase by an additional 4 -fold factor as compared to the glass substrate. Altogether, the results from Fig. 4 show that under $5 \mathrm{~mW} / \mu \mathrm{m}^{2} \mathrm{IR}$ excitation, the temperature increase can be damped from $+17^{\circ} \mathrm{C}$ (glass, illumination from the adhesion layer side) to merely $+1^{\circ} \mathrm{C}$ (sapphire, illumination from opposite side) by properly choosing the substrate and illumination conditions while keeping the same adhesion layer properties. This information can be particularly useful if one has to mitigate the temperature increase in conditions of nano-optical trapping, ${ }^{27,29}$ especially as the adhesion layers for gold cannot be fully avoided for most of the applications.

Lastly, a closer look at the fluorescence and IR transmission time traces show that under conditions of large temperature gains above $+30^{\circ} \mathrm{C}$, additional time-dependent fluctuations appear on top of the shot noise and are anti-correlated between the fluorescence and IR transmission signals (Supporting Information Fig. S3-S4). These fluctuations are related to temperature changes by $\pm 1.5^{\circ} \mathrm{C}$ (Supporting Information Fig. S5) and cannot be ascribed to mechanical drifts of the microscope focus. As irreversible damage to the SNH structure occurs for IR intensities above $5 \mathrm{~mW} / \mu \mathrm{m}^{2}$, the additional noise could be due to some thermally-induced expansion and collapse of the SNH aperture edges, which in turn affects the IR transmission, the local temperature and the fluorescence emission. This also sets an upper limit to about $+40^{\circ} \mathrm{C}$ for the maximum temperature increase reachable with a $13 \mathrm{~nm}$ thick $\mathrm{Cr}$ adhesion layer. 


\section{Conclusions}

While many plasmonics applications cannot avoid using an adhesion layer between the gold film and the

substrate, $, 910,16,18$ the influence of this supplementary adhesion layer remained largely overlooked in the context of the temperature increase of plasmonic nanostructures illuminated by a laser beam. In excellent agreement with numerical simulations, our measurements demonstrate that the adhesion layer plays an essential role in determining the local temperature. If a temperature increase has to be avoided, a thin adhesion layer of $\mathrm{Cr}_{2} \mathrm{O}_{3}$ together with a sapphire substrate provides an effective way to reduce the heat generation and evacuate the heat while maintaining good adhesion properties. On the opposite, if a maximum temperature gain is searched, then a thicker chromium or titanium adhesion layer can significantly increase the temperature change in nanoapertures above $+40^{\circ} \mathrm{C}$. Altogether, these different dedicated designs pave the way for heat-controlled experiments in nanoapertures taking advantage of their well-defined attoliter volumes, high background rejection and accurate local temperature tunability. A broad range of applications can be envisioned, including crystallization and polymerization, ${ }^{37}$ biosensing and enzymatic activity monitoring, ${ }^{23,24}$ DNA and protein nanopore translocation, ${ }^{38-40}$ or thermophoretic trapping. ${ }^{41,42}$

\section{Methods}

\section{Nanohole fabrication}

Cleaned borosilicate or sapphire coverslips of $0.15 \mathrm{~mm}$ thickness are first coated an adhesion layer (either $6 \mathrm{~nm} \mathrm{Ti}, 6 \mathrm{~nm} \mathrm{Cr}, 13 \mathrm{~nm} \mathrm{Cr}$ or $9 \mathrm{~nm} \mathrm{Cr}_{2} \mathrm{O}_{3}$ ) prior to $100 \mathrm{~nm}$ thick gold deposition assisted by electron-beam evaporation (Bühler Syrus Pro 710). Single nanoholes with 300nm diameter are then milled by galliumbased focused ion beam (FEI dual beam DB235 Strata) using $30 \mathrm{keV}$ energy and 10 pA beam current.

\section{Numerical simulations}

Numerical simulations are performed using COMSOL Multiphysics combining "Electromagnetic Waves, Beam Envelopes" and "Heat Transfer in Solids" via the "Electromagnetic Heat Source" module in 3D geometry. The thermal parameters for glass, sapphire, titanium, chromium, chromium oxide, gold and water are taken from the COMSOL material library and assumed to be temperature independent. The 
complex refractive index for $\mathrm{Au}$ (100 nm thick), $\mathrm{Ti}\left(6 \mathrm{~nm}\right.$ thick), $\mathrm{Cr}(13 \mathrm{~nm})$ and $\mathrm{Cr}_{2} \mathrm{O}_{3}(9 \mathrm{~nm})$ are taken from Johnson, Christy ${ }^{45,46}$ and Oldham ${ }^{17}$ for bulk materials (i.e. $\mathrm{n}_{\mathrm{Au}}=0.25846+\mathrm{i} 6.9654, \mathrm{n}_{\mathrm{Ti}}=3.4654+\mathrm{i} 4.0085$, $\mathrm{n}_{\mathrm{Cr}}=3.5408+\mathrm{i} 3.5777, \mathrm{n}_{\mathrm{Cr} 203}=2.0583+\mathrm{i} 0.1123$ at $\left.1064 \mathrm{~nm}\right)$. In the case of chromium oxide, our simulations include a residual $2 \mathrm{~nm}$ thick layer of pure chromium underneath the oxide layer. This supplementary residual amount of chromium was found necessary to match with our experimental observations, as a pure chromium oxide layer would feature even lower absorption (Supporting Information Fig. S1). The refractive index of water, glass and sapphire are taken as 1.33, 1.52, 1.77 respectively. The waist of the incident IR laser beam is $1 \mu \mathrm{m}$, the polarization is linear and the amplitude follows a Gaussian profile.

\section{Optical microscope}

Our inverted confocal microscope contains two overlapping laser beams: one $635 \mathrm{~nm}$ pulsed red laser (Picoquant LDH-P-635) at $80 \mathrm{MHz}$ repetition rate and one continuous wave $1064 \mathrm{~nm}$ infrared laser (Ventus 1064-2W). The red and infrared laser beams are designed for exciting the fluorescence of Alexa Fluor 647 molecules and heating the sample respectively. Both lasers are focused by a high NA microscope objective (Zeiss Plan-Neofluar 40x, NA 1.3, oil immersion). We first estimate the laser spot sizes at the objective focus by recording images of a single nanohole with $300 \mathrm{~nm}$ diameter, which are $0.6 \mu \mathrm{m}$ and $1 \mu \mathrm{m}$ in diameter for the red and infrared lasers respectively. Thus, the power intensity of two lasers could be calculated. The illumination power intensity of the red laser is fixed at $20 \mu \mathrm{W} / \mu \mathrm{m}^{2}$, and we confirmed that no photobleaching and no saturation of the fluorescence of Alexa Fluor 647 molecules occur under this power. The intensity of the infrared laser ranges from 0 to $5 \mathrm{~mW} / \mu \mathrm{m}^{2}$, and corresponds to the conditions used for the plasmonic nano-optical trapping under the damage threshold for the gold film (typically around $50 \mathrm{~mW} / \mu^{2}$ ). Please note that throughout our study, we take into account a $50 \%$ transmission of the microscope objective at $1064 \mathrm{~nm}$, and express the IR illumination intensity at the objective focus (directly incident on the SNH sample).

Alexa Fluor 647 molecules are diluted in phosphate buffered saline solution (PBS) to $200 \mathrm{nM}$ concentration, and a $40 \mu \mathrm{L}$ droplet is deposited on top of the SNH sample. The same microscope objective is used to collect the fluorescence, which is filtered from the backscattered laser light by a set of dichroic mirrors, long pass filters, $30 \mu \mathrm{m}$ confocal pinhole and bandpass filters. Two avalanche photodiodes (Picoquant MPD-5CTC) separated by a 50/50 beam-splitter record the fluorescence photons in the 650$690 \mathrm{~nm}$ spectral range. The fluorescence signals are then sent to a time correlated single photon counting (TCSPC) electronic module (Picoquant Picoharp 300 with PHR 800 router) with time-tagged time-resolved 
(TTTR) option, whose overall temporal resolution (full width at half maximum of the instrument response function) is $100 \mathrm{ps.}$

To measure the infrared transmission at $1064 \mathrm{~nm}$, a low NA microscope objective (Zeiss Plan-Neofluar 10x, NA 0.3) is placed above the sample to collect the IR light transmitted through the SNH. An optical density and a band pass filter $(1064-10 \mathrm{~nm})$ ensure that only the IR light is collected by another avalanche photodiode. All fluorescence and infrared transmission time traces are analyzed with the Symphotime 64 software (Picoquant) enabling to compute the intensity time trace and TCSPC decay histogram for the fluorescence lifetime measurement.

Throughout our work, we carefully checked that our observations upon infrared illumination were fully reversible, i.e. we could retrieve the same results after switching off the $1064 \mathrm{~nm}$ laser than what we found before turning it on. For IR intensities below $5 \mathrm{~mW} / \mu \mathrm{m}^{2}$ at the microscope focus spot, we could not detect any sign of photodamage. Since we are using relatively low laser power of $20 \mu \mathrm{W}$ for red excitation of the fluorescent dyes and since the dyes are constantly diffusing across the nanoaperture (providing a constant source of "fresh" fluorescent molecules), we did not monitor any sign of fluorescence saturation or photobleaching.

\section{Temperature measurement based on fluorescence lifetime}

The temperature measurements inside the SNH follow the fluorescence lifetime approach introduced in ref ${ }^{36}$ and briefly summarized here. The non-radiative decay rate of many fluorescent molecules, such as Alexa Fluor 647 used in this article, is very sensitive to the temperature change. We calibrated the change in the non-radiative decay rate of Alexa Fluor 647 in a homogeneous water medium with temperature increasing above the room temperature $\left(21^{\circ} \mathrm{C}\right)$ :

$$
\Delta T=91.31-91.22 \times\left(\frac{\Gamma_{n r a d}\left(21^{\circ} \mathrm{C}\right)}{\Gamma_{n r a d}(T)}\right)^{0.42}
$$

where $\Gamma_{\text {nrad }}\left(21^{\circ} \mathrm{C}\right)$ and $\Gamma_{\text {nrad }}(T)$ are the non-radiative decay rates at room temperature and any temperature above $21^{\circ} \mathrm{C}$. By considering the radiative rate and plasmonic loss rate constant when the plasmonic nanohole is introduced, ${ }^{36}$ the non-radiative decay rate inside the nanohole can be determined by: 


$$
\frac{\Gamma_{\text {nrad }}(T)}{\Gamma_{\text {nrad }}\left(21^{\circ} \mathrm{C}\right)}=1+\frac{1}{\Gamma_{n \text { rad }}\left(21^{\circ} \mathrm{C}\right)}\left(\frac{1}{\tau_{T}{ }^{*}}-\frac{1}{\tau_{21^{\circ} \mathrm{C}}{ }^{*}}\right)
$$

where $\tau_{T}{ }^{*}$ and $\tau_{211^{\circ} \mathrm{C}}{ }^{*}$ are the fluorescence lifetime measured in the nanohole at any temperature above $21^{\circ} \mathrm{C}$ and room temperature which are expressed in nanoseconds. $\Gamma_{n r a d}\left(21^{\circ} \mathrm{C}\right)$ is used as $0.67 \mathrm{~ns}^{-1}$ by knowing the 1.0 ns lifetime and 33\% quantum yield of Alexa Fluor 647 at room temperature. By combining equations (1) and (2), we finally deduce the relationship between the temperature increase in a single nanohole and the fluorescence lifetime reduction:

$$
\Delta T=91.31-91.22 \times\left[1+\frac{1}{0.67}\left(\frac{1}{\tau_{T}{ }^{*}}-\frac{1}{\tau_{21^{\circ} C^{*}}}\right)\right]^{-0.42}
$$

Equation (3) provides the way to measure the temperature increase $\Delta T$ for any SNH structure by simply measuring the Alexa Fluor 647 fluorescence lifetime inside the SNH with $\left(\tau_{T}{ }^{*}\right)$ and without $\left(\tau_{21}{ }^{\circ}{ }^{*}\right)$ the heating infrared laser. The experimental errors for the temperature measurement based on the fluorescence lifetime mainly stem from the uncertainties induced by the noise during the curve-fitting process, and are about $7 \%$ of the temperature increase.

An advantage of this approach is that it only requires two fluorescence lifetime measurements, which do not depend on the local concentration of fluorescence molecules and can even be performed if the sample is slightly defocused on the microscope. Let us also point out that we have carefully checked in ref. ${ }^{36}$ that the $1064 \mathrm{~nm}$ infrared beam alone does not noticeably influence the Alexa Fluor 647 photophysics for IR intensities below $5 \mathrm{~mW} / \mu \mathrm{m}^{2}$.

The fluorescence lifetime is obtained by fitting the fluorescence decay histograms using a LevenbergMarquard optimization performed on a commercial software (PicoQuant SymPhoTime 64). The fitting model is implemented by an iterative reconvolution including the instrument response function (IRF) with more than $96 \%$ of all detected photons being included. The fluorescence decay curves of Alexa Fluor 647 in the nanoapertures are well fitted with a biexponential function. The first short lifetime component is fixed at 150 ps to account for the non-radiative losses into the metal and has a moderate influence. The dominating second lifetime component provides the relevant fluorescence lifetime.

\section{Supporting Information}

Linear relationship between thickness and infrared absorption, comparison between $\mathrm{Cr}$ and $\mathrm{Ti}$ adhesion layers of $6 \mathrm{~nm}$ thickness, fluorescence and infrared transmission time traces analysis. 


\section{Funding Sources}

This project has received funding from the European Research Council (ERC) under the European Union's Horizon 2020 research and innovation programme (grant agreement No 723241 TryptoBoost) and from the Agence Nationale de la Recherche (ANR) under grant agreement ANR-17-CE09-0026-01 and ANR-18CE42-0013.

\section{Conflict of Interest}

The authors declare no competing financial interest.

\section{Acknowledgements}

The authors thank Olivier Hector for help with the thin film deposition.

\section{References}

1 L. Novotny and B. Hecht, Principles of Nano-Optics, Cambridge University Press, 2012.

2 M. L. Brongersma, N. J. Halas and P. Nordlander, Nat. Nanotechnol., 2015, 10, 25-34.

3G. Baffou and R. Quidant, Laser Photonics Rev., 2013, 7, 171-187.

4 G. Baffou, R. Quidant and F. J. García de Abajo, ACS Nano, 2010, 4, 709-716.

5 G. Baffou, Thermoplasmonics, Cambridge University Press, 2017.

6J. Qiu and W. D. Wei, J. Phys. Chem. C, 2014, 118, 20735-20749.

7 G. Baffou and R. Quidant, Chem. Soc. Rev., 2014, 43, 3898-3907.

8 L. Jauffred, A. Samadi, H. Klingberg, P. M. Bendix and L. B. Oddershede, Chem. Rev., 2019, 119, 80878130.

9 M. Todeschini, A. Bastos da Silva Fanta, F. Jensen, J. B. Wagner and A. Han, ACS Appl. Mater. Interfaces, 2017, 9, 37374-37385.

10 W. M. Abbott, C. P. Murray, C. Zhong, C. Smith, C. McGuinness, E. Rezvani, C. Downing, D. Daly, A. K. Petford-Long, F. Bello, D. McCloskey and J. F. Donegan, ACS Appl. Mater. Interfaces, 2019, 11, 7607-7614. 
12 H. Neff, W. Zong, A. M. N. Lima, M. Borre and G. Holzhüter, Thin Solid Films, 2006, 496, 688-697.

13 B. A. Sexton, B. N. Feltis and T. J. Davis, Sens. Actuators Phys., 2008, 141, 471-475.

14 D. T. Debu, P. K. Ghosh, D. French and J. B. Herzog, Opt. Mater. Express, 2017, 7, 73-84.

15 M. Najiminaini, F. Vasefi, B. Kaminska and J. J. L. Carson, Opt. Express, 2011, 19, 26186-26197.

16 H. Aouani, J. Wenger, D. Gérard, H. Rigneault, E. Devaux, T. W. Ebbesen, F. Mahdavi, T. Xu and S. Blair, ACS Nano, 2009, 3, 2043-2048.

17 X. Jiao, J. Goeckeritz, S. Blair and M. Oldham, Plasmonics, 2009, 4, 37-50.

18 T. Siegfried, Y. Ekinci, O. J. F. Martin and H. Sigg, ACS Nano, 2013, 7, 2751-2757.

19 N. Djaker, R. Hostein, E. Devaux, T. W. Ebbesen, H. Rigneault and J. Wenger, J. Phys. Chem. C, 2010, 114, 16250-16256.

20 F. Colas, D. Barchiesi, S. Kessentini, T. Toury and M. L. de la Chapelle, J. Opt., 2015, 17, 114010.

21 B. J. Roxworthy and K. C. Toussaint, Opt. Express, 2012, 20, 9591-9603.

22 H. Ma, P. Tian, J. Pello, P. M. Bendix and L. B. Oddershede, Nano Lett., 2014, 14, 612-619.

23 M. J. Levene, J. Korlach, S. W. Turner, M. Foquet, H. G. Craighead and W. W. Webb, Science, 2003, 299, 682-686.

24 P. Zhu and H. G. Craighead, Annu. Rev. Biophys., 2012, 41, 269-293.

25 D. Punj, P. Ghenuche, S. B. Moparthi, J. de Torres, V. Grigoriev, H. Rigneault and J. Wenger, Wiley Interdiscip. Rev. Nanomed. Nanobiotechnol., 2014, 6, 268-282.

26 M. L. Juan, M. Righini and R. Quidant, Nat. Photonics, 2011, 5, 349-356.

27 M. L. Juan, R. Gordon, Y. Pang, F. Eftekhari and R. Quidant, Nat. Phys., 2009, 5, 915-919.

28 Y. Pang and R. Gordon, Nano Lett., 2011, 11, 3763-3767.

29 Y. Pang and R. Gordon, Nano Lett., 2012, 12, 402-406.

30 J. Berthelot, S. S. Aćimović, M. L. Juan, M. P. Kreuzer, J. Renger and R. Quidant, Nat. Nanotechnol., 2014, 9, 295-299.

31 R. A. Jensen, I.-C. Huang, O. Chen, J. T. Choy, T. S. Bischof, M. Lončar and M. G. Bawendi, ACS Photonics, 2016, 3, 423-427.

32 M. Ghorbanzadeh, S. Jones, M. K. Moravvej-Farshi and R. Gordon, ACS Photonics, 2017, 4, 11081113.

33 A. Kotnala and R. Gordon, Nano Lett., 2014, 14, 853-856.

34 P. Mestres, J. Berthelot, S. S. Aćimović and R. Quidant, Light Sci. Appl., 2016, 5, e16092. 
35 Z. Xu, W. Song and K. B. Crozier, ACS Photonics, 2018, 5, 2850-2859.

36 Q. Jiang, B. Rogez, J.-B. Claude, G. Baffou and J. Wenger, ACS Photonics, 2019, 6, 1763-1773.

37 T. Sugiyama, K. Yuyama and H. Masuhara, Acc. Chem. Res., 2012, 45, 1946-1954.

38 F. Nicoli, D. Verschueren, M. Klein, C. Dekker and M. P. Jonsson, Nano Lett., 2014, 14, 69176925.

39 X. Shi, D. V. Verschueren and C. Dekker, Nano Lett., 2018, 18, 8003-8010.

40 D. V. Verschueren, S. Pud, X. Shi, L. De Angelis, L. Kuipers and C. Dekker, ACS Nano, 2019, 13, 61-70.

41 J. C. Ndukaife, A. V. Kildishev, A. G. A. Nnanna, V. M. Shalaev, S. T. Wereley and A. Boltasseva, Nat. Nanotechnol., 2016, 11, 53-59.

42 M. Braun and F. Cichos, ACS Nano, 2013, 7, 11200-11208.

43 K. Wang, E. Schonbrun, P. Steinvurzel and K. B. Crozier, Nat. Commun., 2011, 2, 469.

44 G. Baffou, C. Girard and R. Quidant, Phys. Rev. Lett., 2010, 104, 136805.

45 P. B. Johnson and R. W. Christy, Phys. Rev. B, 1972, 6, 4370-4379.

46 P. B. Johnson and R. W. Christy, Phys. Rev. B, 1974, 9, 5056-5070. 


\title{
Supporting Information for \\ Adhesion Layer Influence on Controlling the Local Temperature in Plasmonic Gold Nanoholes
}

\author{
Quanbo Jiang, Benoît Rogez, Jean-Benoît Claude, Antonin Moreau, Julien Lumeau, Guillaume Baffou, \\ Jérôme Wenger \\ Aix Marseille Univ, CNRS, Centrale Marseille, Institut Fresnel, 13013 Marseille, France
}

This document contains the following supporting information:

S1. Linear relationship between thickness and infrared absorption

S2. Comparison between $\mathrm{Cr}$ and $\mathrm{Ti}$ adhesion layers of $6 \mathrm{~nm}$ thickness

S3. Fluorescence and infrared transmission time traces analysis

\section{S1. Linear relationship between thickness and infrared absorption}

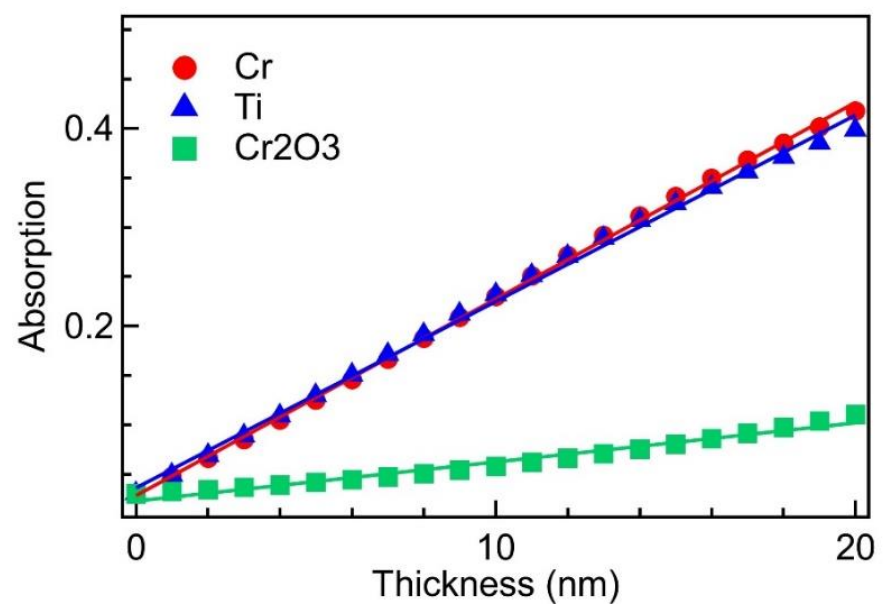

Figure S4. Analytical simulation of the $1064 \mathrm{~nm}$ infrared absorption as a function of the thickness of adhesion layers underneath a $100 \mathrm{~nm}$ thick gold film. The calculations are performed using Fresnel coefficients for multilayer systems. The simulation data of chromium ( $\mathrm{Cr}$, red), titanium ( $\mathrm{Ti}$, blue) and chromium oxide $\left(\mathrm{Cr}_{2} \mathrm{O}_{3}\right.$, green) follow a quasi-linear dependence with the layer thickness for the thickness range below $20 \mathrm{~nm}$. Additionally, the $\mathrm{Cr}$ and Ti films feature a similar IR absorption which leads to a comparable temperature increase for the same thickness. 


\section{S2. Comparison between $\mathrm{Cr}$ and $\mathrm{Ti}$ adhesion layers of $6 \mathrm{~nm}$ thickness}

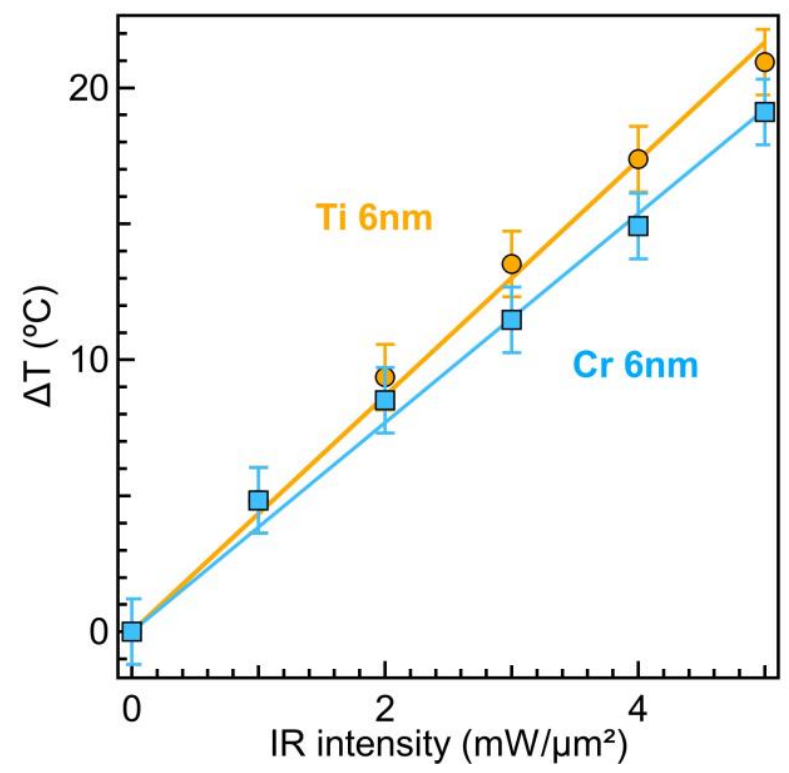

Figure S2. Temperature increase as a function of the infrared intensity at $1064 \mathrm{~nm}$ measured on a $300 \mathrm{~nm}$ diameter gold nanoaperture for chromium and titanium adhesion layers of similar $6 \mathrm{~nm}$ thickness. The experimental conditions are identical to Fig. 2 of the main document. Numerical simulations indicate that $\mathrm{Cr}$ and Ti films share a similar IR absorption (Fig. S1). Here our experimental data confirms that both $\mathrm{Cr}$ and $\mathrm{Ti}$ adhesion layers (of same thickness) lead to a comparable temperature increase within the experimental uncertainties.

\section{S3. Fluorescence and infrared transmission time traces analysis}

Having established how to control the local temperature inside a $\mathrm{SNH}$, we take a closer look at the fluorescence and IR transmission signals under conditions of large temperature gains up to $+40^{\circ} \mathrm{C}$. First, we confirm that the optical microscope setup is stable, leading to a steady fluorescence time trace where the noise fluctuations are dominated by shot noise (Fig. S3a). We also check that the transmitted IR signal is stable in the absence of gold structure (Fig. S3b). The situation becomes clearly different when a SNH is illuminated, leading to a temperature raise of $+32^{\circ} \mathrm{C}$ (Fig. S3c,d). Additional fluctuations appear on top of the shot noise with a time scale of a few seconds, and are anti-correlated between the fluorescence and IR transmission signals. More time traces for increasing IR intensities are shown in Fig. S4. 

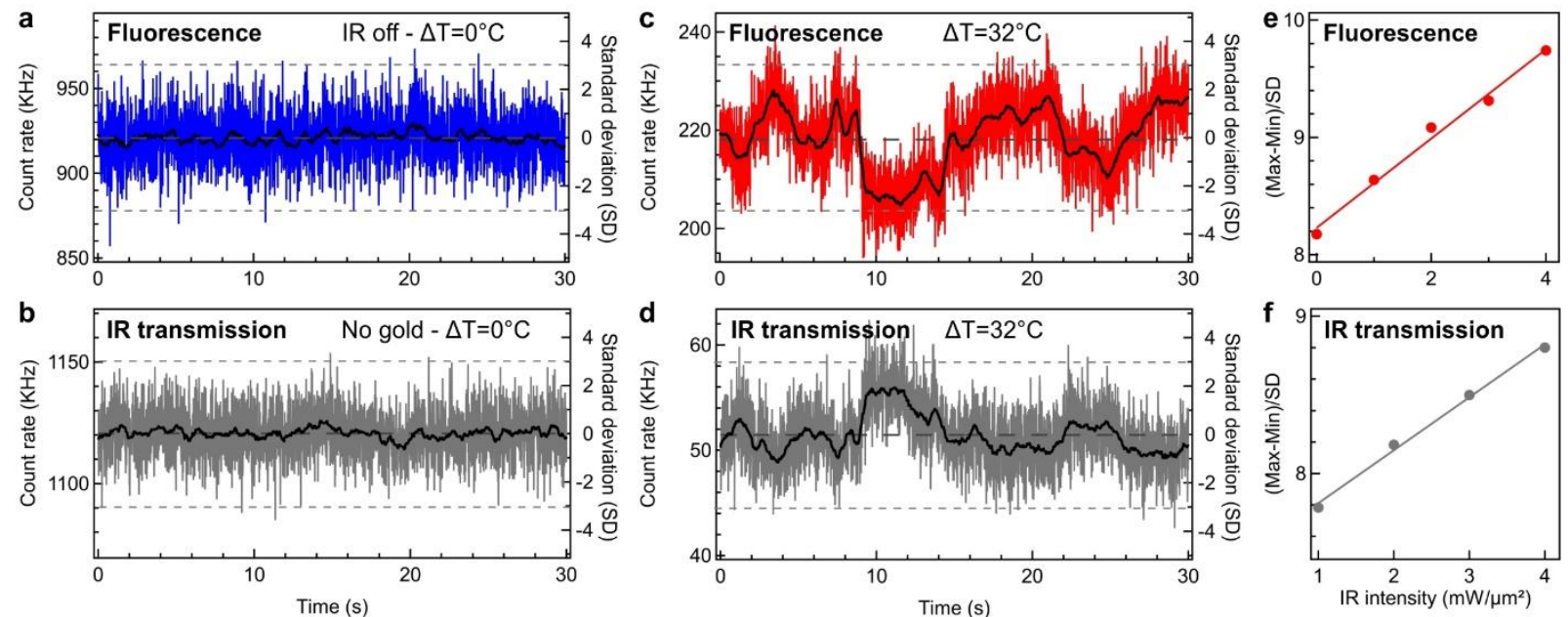

Figure S3. Time traces of fluorescence intensity and infrared transmission in a $300 \mathrm{~nm}$ diameter SNH with $13 \mathrm{~nm} \mathrm{Cr}$ adhesion layer. (a) Alexa Fluor 647 fluorescence intensity on a SNH with no heating infrared beam: the signal is stable and within \pm 3 standard deviation (SD) as indicated by the horizontal dashed lines. The thin blue trace corresponds to a binning time of $10 \mathrm{~ms}$ while the thick black trace is smoothed over a 500 ms period. (b) Infrared transmission when no metal film is present, again the trace is stable and within \pm 3 SD. (c,d) Fluorescence and IR transmission when the SNH is illuminated at $4 \mathrm{~mW} / \mu \mathrm{m}^{2} \mathrm{IR}$ intensity, leading to an average temperature increase of $+32^{\circ} \mathrm{C}$. Additional fluctuations are clearly visible on top of the shot noise, and are anticorrelated between fluorescence and IR transmission. (e,f) Analysis of the fluctuation amplitude normalized by the standard deviation defined as (max-min)/SD for the fluorescence (e) and IR transmission ( $f$ ) time traces as a function of the IR intensity.

We quantify the fluctuations amplitude by recording the peak-to-peak amplitude (defined as the difference between the maximum and minimum values found for a $60 \mathrm{~s}$ trace), and normalize it by the standard deviation (SD) calculated from the most stable (flat) parts within $2 \mathrm{~s}$ (200 points) in each time trace. For a shot-noise limited Gaussian statistical distribution, the peak-to-peak values should remain within 6 to 8 times the SD. However, our experimental observations clearly deviate from this range and increase with the IR power (Fig. S3e,f), indicating the presence of a supplementary source of fluctuations. Specific time intervals can be selected on the fluorescence time trace to estimate the fluorescence lifetime and local temperature during events corresponding to high or low amplitude fluctuations (Fig. S5). When the fluorescence intensity is clearly below the average level (Fig. S5a, red selected part), the fluorescence lifetime is shorter, indicating a higher temperature of $+32.6^{\circ} \mathrm{C}$. Reversely, when the fluorescence intensity is above the average level (Fig. S5a, blue selected part), the fluorescence lifetime is longer and the 
temperature is lower with a value of $+31.0^{\circ} \mathrm{C}$. The $1.6^{\circ} \mathrm{C}$ temperature difference between the cases is moderate, but significantly above the experimental noise level. Let us also stress that the fluorescence lifetime measurement depends only on the arrival time of the fluorescence photons. This measurement is largely independent of the intensity of the fluorescence signal, therefore the fluctuations observed cannot be ascribed to mechanical drifts of the microscope focus. We have checked that the fluorescence properties of the molecules is not affected by the IR illumination alone up to values of $80 \mathrm{~mW} / \mathrm{\mu m}^{2}{ }^{2}{ }^{\mathrm{s}}$ Currently, our observations indicate the occurrence of a change in IR transmission, leading to a modification of the local temperature and in turn an alteration of the fluorescence lifetime and intensity of Alexa Fluor 647 molecules. The nature and origin of the IR transmission fluctuations remain unclear. It should be noted than at $5 \mathrm{~mW} / \mu \mathrm{m}^{2}$ we are approaching the damage threshold for the gold film on a 13 $\mathrm{nm} \mathrm{Cr}$ layer. When we performed experiments at values higher than this level, we could not retrieve the initial values anymore when the IR intensity was reduced. This indicates the occurrence of irreversible damage to the SNH structure, and sets an upper limit for the maximum temperature increase reachable. Therefore, we believe that the IR transmission fluctuations could be due to some thermally-induced expansion or collapse of the SNH aperture edges, which in turn induces the modifications of the local temperature and fluorescence properties. We have also checked that convection fluxes in the water medium do not play a significant role in our observations. Similar results were obtained with two different liquid heights above the SNH: one height of a few micrometers (where convection is prohibited) and one of several hundreds of micrometers (where convection may occur). 

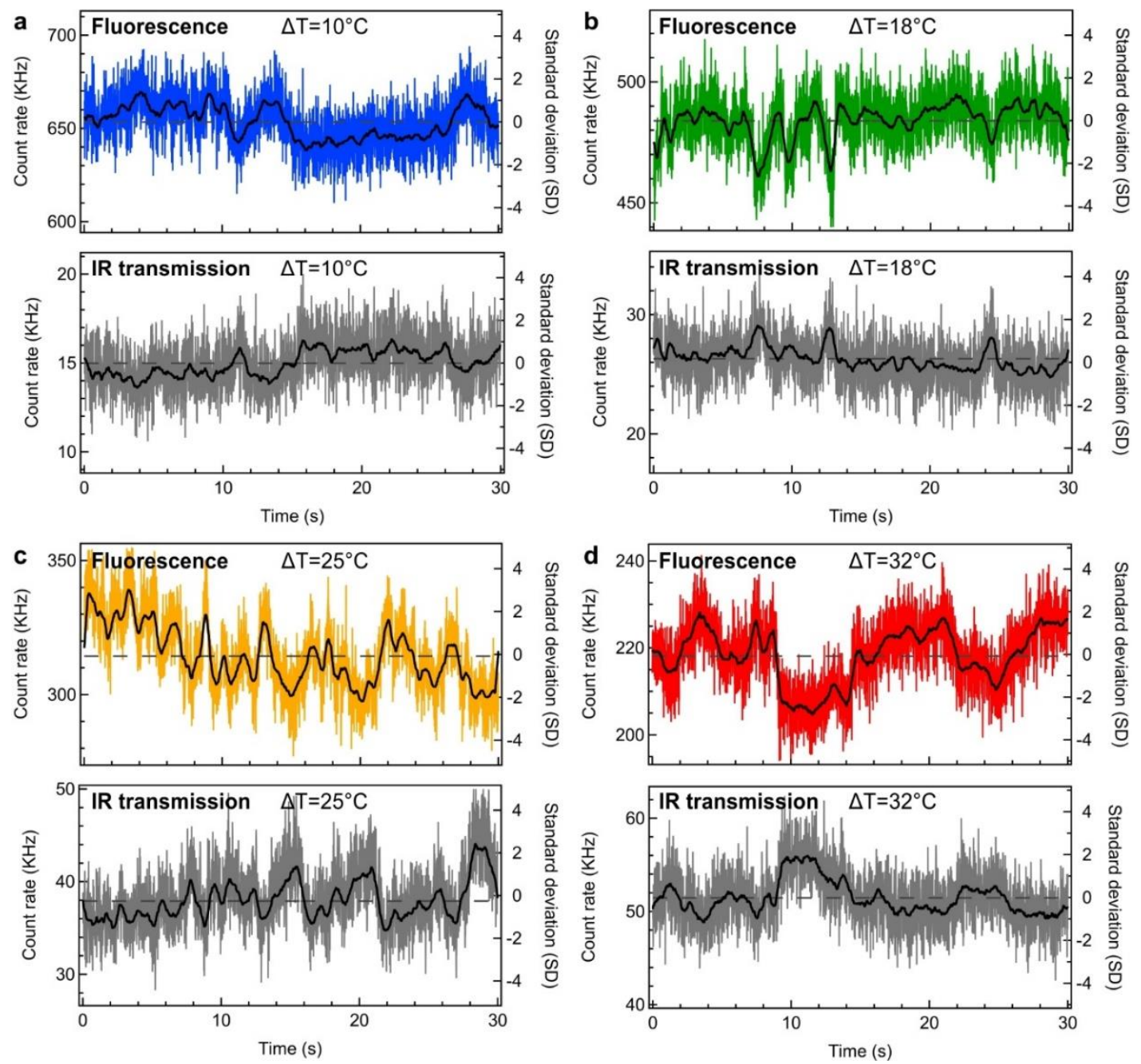

Figure S4. Time traces of fluorescence intensity and infrared transmission for a $300 \mathrm{~nm}$ diameter SNH with $13 \mathrm{~nm} \mathrm{Cr}$ adhesion layer. Through (a-d) the IR intensity increases from 1 to $4 \mathrm{~mW} / \mu \mathrm{m}^{2}$. The temperature increase corresponds to $+10^{\circ} \mathrm{C},+18^{\circ} \mathrm{C},+25^{\circ} \mathrm{C}$ and $+32^{\circ} \mathrm{C}$ respectively. The thin color or gray traces correspond to a $10 \mathrm{~ms}$ binning time while the thick black traces are averaged over a $500 \mathrm{~ms}$ period. The dashed horizontal lines indicate the average value and the right vertical axis is normalized by the standard deviation for each trace. 

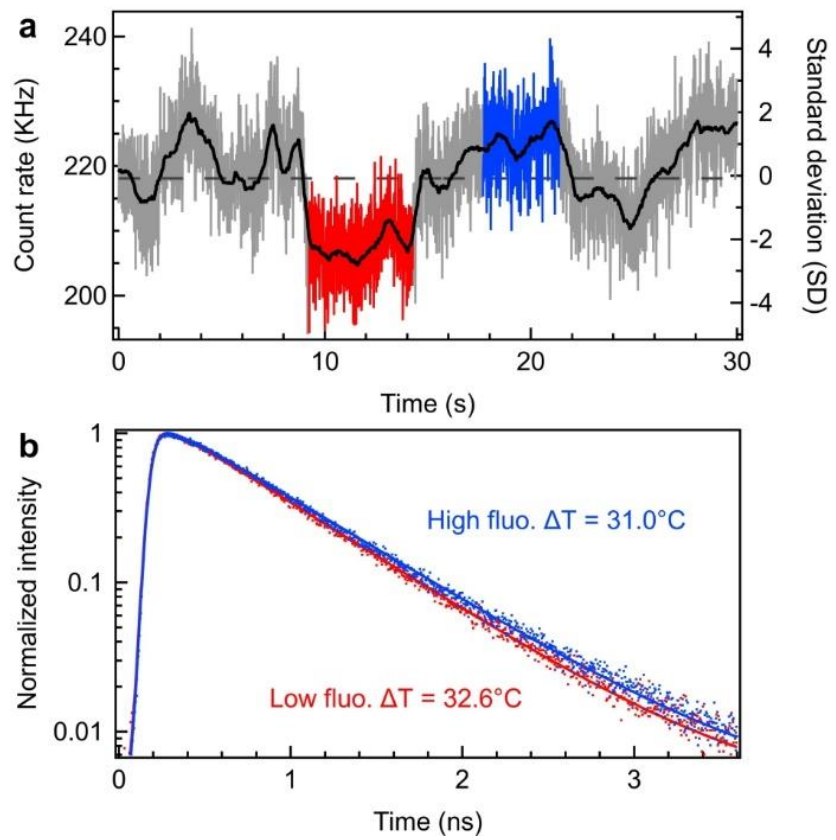

Figure S5. Fluorescence lifetime measured on a selected time interval corresponding to high/low fluctuations of the fluorescence intensity. (a) Fluorescence time trace (same as Fig. S2c). The lower (red) and higher fluorescence (blue) parts are selected for the partial lifetime measurement instead of the average of the overall time trace. (b) Normalized fluorescence decay curves of Alexa Fluor 647 solution corresponding to the red and blue parts in (a). The temperature gains extracted from the measured lifetimes are indicated on the graph.

\section{Refererence}

(S1) Q. Jiang, B. Rogez, J.-B. Claude, G. Baffou and J. Wenger, ACS Photonics, 2019, 6, 1763-1773. 